\title{
Leukemia case triggers tighter gene-therapy controls
}

A hastily convened advisory committee to the US Food and Drug Administration (FDA) ruled last month that gene-therapy trials of the kind that led to a leukemia-like illness in a French patient, and which were halted because of that case, should now continue. However, there must be increased monitoring for adverse events in patients, and they must receive modified informed consent forms to explain the chances of this side effect occurring. "One adverse event, as serious as it is, in the context of the whole field is not enough to put all programs on hold," said committee chair Daniel Salomon of The Scripps Research Institute.

Trials of gene therapy for severe combined immune deficiency (SCID), a fatal disease caused by deficiencies in immune-cell function, were put on hold in the United States and France following the report that a three-yearold patient treated by Alain Fischer and colleagues at the Necker Hospital in Paris had developed leukemia after being treated with a retroviral vector. Advisors to the British government decided against halting two similar trials in the United Kingdom, but recommended closer monitoring.

In this type of gene therapy, retroviral vectors are used to treat ex vivo a patient's bone marrow stem cells, which give rise to functioning blood cells. The FDA committee met on October 10 to ask whether there were "additional data or measures that clinical investigators need to provide before future and present clinical trials in SCID patients should proceed in the US." They agreed that monitoring of abnormal activity of certain cells and of the sites in the genome where the retroviral vector integrates should be conducted on an ongoing basis during SCID trials. Salomon dismissed suggestions that this type of monitoring is too cumbersome and expensive. "It needs to be done," he said.

In addition, informed consent forms for all trials that use retroviruses should be changed to warn patients explicitly that a case of leukemia occurred in a SCID gene-therapy trial and that the event was related to the treatment.

Although the French patient is the first gene-therapy recipient to develop cancer, scientists have long warned of the possible risk. Retroviruses integrate at random into the genome and have been shown to cause tumors in mice. Stuart Orkin of Harvard Medical School reminded the panel of a 1995 National Institutes of Health report that he co-authored, which stated that "multiple integration events resulting from repeated administration of large doses of retroviruses theoretically pose a risk for leukemic transformation." Others in the audience agreed that this concern has been communicated to patients but that the extent of the risk is not known. "We don't know the risk," said Orkin. "The most prudent thing is to watch carefully."

The FDA halted the three trials in the United States that were most similar to the French trial, but it also plans to contact the sponsors of the 150 or so ongoing and completed gene-therapy trials that use retroviral vectors so that patients and their survivors can be notified. Abbey Meyers, president of the National Organization for Rare Disorders, criticized the FDA for not yet having created a registry of genetherapy trials as mandated by Congress eight years ago. Philip Noguchi, acting chair of the FDA's Office of Cell, Tissue and Gene Therapies, agreed that the registry is important and admitted, "but we don't have it yet."

Fischer's trial in France included 11 patients with X-linked SCID_the most common and severe form of the disease. The scientists were hailed in 2000 for the first successful application of gene therapy. The therapy was successful in ten of the patients, including patient no. 4, a boy treated at one month of age. But although he initially responded well, 30 months after treatment the boy developed an excess of a particular type of $\mathrm{T}$ cell. This August, he began a regime of chemotherapy, and the trial was suspended.

Using a new PCR-based technique, Christof Von Kalle of Cincinnati Children's Hospital (and formally at the University of Freiburg Medical Center) discovered that the retroviral vector used in the French trial had inserted into more than 40 sites in the genome of different repopulating cells. In the T-cell clone that grew abnormally, the vector had inserted in the LMO-2 oncogene on chromosome 11a gene originally identified as a breakpoint of a translocation that causes a type of T-cell leukemia. The retrovirus insertion appeared to have caused increased expression of the gene. Kalle detected increased activity of the T-cell clone carrying the LMO-2 integration in blood samples taken from the boy as early as 13 months after treatment, well before he showed any clinical symptoms.

From the data, Orkin, an expert in leukemia, concluded: "Insertion of a retrovirus in [the LMO-2] site is likely to be related to the outcome and phenotype [in the patient]." However, Orkin and other scientists agreed that expression of $L M O-2$ by itself was probably not sufficient for leukemia but that a second event was required, such as inflammation or mutation in another oncogene or tumor suppressor gene, for cancer to ensue. Kalle and Fischer are now trying to identify this other event.

Another question that Fischer is trying to address surrounds the possibility that the boy had a genetic predisposition to leukemia, as there have been two childhood cancers in the family, including a cancer in the patient's sister.

The only alternative treatment for SCID is bone marrow transplantation. This approach has an almost $100 \%$ success rate if the donor is a perfect match for the recipient, but drops to less than $80 \%$ for partially matched donors and recipients; and there are some longterm problems, including incomplete B-cell function and possible graft-versus-host disease. The people enrolled in the French gene-therapy trial did not have HLA-identical donors. Currently, there are 636 gene-therapy trials completed, ongoing or pending worldwide. Laura Bonetta, Bethesda 